\title{
Augmented Reality Mobile Application Co-Design Experience: Delineating the Nuances from the Lens of a System Developer
}

\author{
Mas Nida Md. Khambari ${ }^{1}$, Fadzil Saleh Mohamad Rofie ${ }^{1}$ \\ ${ }^{1}$ Faculty of Educational Studies, Universiti Putra Malaysia, Serdang, Selangor, Malaysia \\ Correspondence: Mas Nida Md. Khambari, Faculty of Educational Studies, Universiti Putra Malaysia, 43400 \\ UPM Serdang, Selangor, Malaysia Tel: 603-9769-8178. E-mail: khamasnida@upm.edu.my
}

Received: September 3, 2021

Accepted: September 28, 2021 Online Published: October 16, 2021

doi:10.5539/ass.v17n11p198

URL: https://doi.org/10.5539/ass.v17n11p198

\begin{abstract}
With the rapid development of educational tools, co-design has been on the rise. Co-design is instrumental in successful product development as it merges two key perspectives, namely consumers' insights and professionals' knowledge. The roles of users, designers, and developers are now blurred as educators have begun to construct their own tools for teaching based on problems and ideas conceived in their classrooms, thereby assuming roles as designers and to some extent, as developers. A more common practice in recent days is educators' co-designing of tools with system developers to achieve their targeted goal. This study attempted to explore the process of co-designing an augmented reality (AR) mobile application and the role of a system developer in mediating the design process with non-designers, namely academic members of the Faculty of Medicine and Health Sciences, Universiti Putra Malaysia. This study further aimed to delineate the nuances of the AR mobile application co-design experience from the perspective of the system developer. Semi-structured interviews, observations, and document analyses were conducted to examine the detailed process of co-designing and developing the AR application as well as to understand the developer's interaction with the consumers. The main findings of this study suggest that the waterfall model of the Software Development Life Cycle was in use during the co-design process. This cycle generally involves five stages, namely (i) planning, (ii) analysis, (iii) design, (iv) implementation, and (v) maintenance, which are iterative in nature. The designer's role in mediating the co-design process encompassed balancing their knowledge and experience with the needs of consumers that do not necessarily match the designer's expectations. This was achieved through (i) precise communication, (ii) commitment to the delivery and quality of the AR mobile application, as well as to building cohesive working relationships, and (iii) motivation to work with co-designers during the development process. The findings shed light on the value of co-design and the complex role of designers in mediating the design process with non-designers, which when accounted for, can lead to more feasible project development.
\end{abstract}

Keywords: augmented reality mobile application, co-design experience, Software Development Life Cycle, Waterfall Model, communication, commitment, motivation

\section{Introduction}

Exponentially emerging technologies have brought significant changes to classrooms, transforming the landscape of education and teaching and learning practices. Several educational tools have been built from the ground up as a result of classroom research carried out by educators to optimize technologies in response to their learners' needs. Consequently, the role of users, designers, and developers have blurred as educators are now constructing their own tools for teaching based on problems and ideas conceived in their classrooms, thereby assuming roles as designers and to some extent, as developers.

This study presents the scenario of a designer who, with a group of academicians from the Faculty of Medicine and Health Sciences (FMHS), Universiti Putra Malaysia (UPM), co-designed and developed an augmented reality (AR) mobile application for the teaching and learning of the Central Nervous System. Medical education nowadays leverages three-dimensional visualization software, mobile applications, and virtual cadavers for the teaching and learning of medical courses, especially those that involve human anatomy (Darras et al., 2019; Van Dijck, 2000). These technologies do not only provide a more realistic and advanced representation of the human body but can also be used and reused without limitations. In tandem with efforts to integrate digital technologies in the teaching and learning process, using AR technology benefits instructors and learners in understanding core 
concepts via three-dimensional visualization. To successfully develop this AR mobile application, the system developer and academicians came together to co-design the system.

Co-design is instrumental in successful product development as it merges two key perspectives, namely consumers' insights and professionals' knowledge (Trischler et al., 2017). According to Sanders and Stappers (2014), co-design is the collective creativity of collaborating parties (who comprise designers and non-designers) in a design development process. Co-design has become a more common practice in recent days as designers are moving towards human-centric design (Sanders \& Stappers, 2014). This shift has opened up opportunities for educators to co-design their tools with system developers to achieve their targeted goals. Some have suggested that such collaborative work can beget myriad innovative solutions for complex issues, reduce the duplication of efforts through the concerted initiatives of multiple individuals, and promote the efficient management of financial resources (Boyd \& Peters, 2009; Brown et al., 2012). The study by Warburton et al. (2008) further confirmed that the benefits of collaborative work extend to the creation of a higher quality program and a more comprehensive system.

Even though Trischler et al. (2017) suggested that co-design teams that work collaboratively to develop design concepts can achieve higher user benefit and novelty, they also found that such teams can result in lower feasibility. This means that the designer may have to compromise the feasibility of the system to fulfill the needs of consumers. Conflicting insights and values are also common during co-design. Having pointed this out some time ago, Ceraulo (2005) mentioned that unresolved issues caused by differences between designers and their consumers may affect the quality and function of a system. Thus, finding harmony within dissonance is the major challenge of the co-design practice (Molnar \& Palmas, 2021; Mulvale et al., 2020).

Molnar and Palmas (2021) identified the co-design process as a negotiation-based search for settlements in a way that may not necessarily solve conflicts but may suspend the potential effects of a conflict should it happen between the system developer and the consumers. Negotiations and diplomacy are key to the success of co-design; as such, the process of co-design is typically conducted within a "designerly peacemaking" setting (Molnar \& Palmas, 2021, p. 1). To ensure co-design success, Trischelr et al. (2018) recommended system developers to carefully select customers, assemble designer teams, and manage intra-team dynamics.

It is evident in the literature that the cohesive relationship between co-designers (i.e., system developers and consumers) must be understood, as the co-design process faces several challenges in this regard. A study by Marek et al. (2015) indicated that although collaboration can be seen as an effective means of improving work culture issues, successful collaboration is still difficult to achieve; rather, failure is more prevalent. In a more recent study by Dimopoulos-Bick et al. (2018), it was found that challenges in co-design usually revolve around power and equity for equal say, commitment to the process, methods of gathering experiences that are usually not interpreted or contextualized collaboratively, design improvements, and implementation. The authors added that limited resources, such as time and money, and a traditional hierarchical decision-making process may impact co-design projects as well, to the extent that they can stall implementation efforts.

Adding to these findings, Jones et al. (2020) outlined several forms of consumer participation in co-design, including survey questionnaires, interviews (face-to-face, over-the-phone, or digital), interactive workshops or meetings (face-to-face or digital), design charrettes (face-to-face or digital with bigger groups), design jams (face-to-face or digital with smaller groups), crowdsourcing (open calls to a larger group), hackathon (digital events with large groups), and ethnographic approaches like observations. Although several studies have reported the many benefits of collaborative work in fostering effective co-design practice, the aforesaid forms of participation in co-design come with their own challenges. Among others, an approach may be intrusive and make consumers reluctant to give feedback. Some techniques may also be unable to appropriately measure the breadth and depth of the problem, subject to social biases, or lack harmony when there is dissonance between designers' knowledge and consumers' insights and needs (Jones et al., 2020; Molnar \& Palmas, 2021; Mulvale et al., 2020).

Despite these issues, Ashkenas (2015) highlighted that well-meaning cooperation is the key to collaborative behavior. Marek et al. (2015) echoed that successful collaboration can still be achieved if the factors of communication, function, leadership, process, members, resource, and context are fulfilled. Furthermore, Keys et al. (2016) concluded that organizational dynamics and a variety of communication strategies are the most common elements of successful inter-profession collaboration. Molnar and Palmas (2021) also recommended negotiation and diplomacy as essential elements of successful co-design collaboration between system developers and consumers.

In another study, a clear understanding of roles and information design were identified as success factors in 
easing into the culture of co-operating and providing solutions to common communication problems (Waller et al., 2016). This has long been recommended by Yousuf et al. (2008), who asserted that more effort needs to be focused on communication and organization in team meetings to ensure individuals are aware of their roles and responsibilities and improve their commitment towards achieving expected goals.

Saraiva and Iglesias (2016) revealed evidence that time constraints and time-related variables may affect people's roles in terms of whether they want to cooperate or compete in organizations. This is due to the social dilemma that forces individuals to choose between a personal need and group goals; often, they need to sacrifice one for the other. This is consistent with the study by Van Lange et al. (2013) that examined social dilemmas, the need for people to cooperate, and how such cooperation can be improved and maintained in groups.

One of the most significant current discussions within the field of co-design is team motivation. Team motivation can be defined as the coordination of collective efforts with high intensity to achieve team goals (Grant \& Shin, 2012). The growth of literature on team motivation is not surprising considering the vast amount of extant research on team organization and work teams (Hollenbeck et al., 2012). For example, Zhu et al. (2016) focused on the effects of intrinsic and extrinsic motivation on the creativity of employees. They suggested that collaborative and competitive climates influence employees' creativity and improve their motivation.

From another perspective, Dent-Spargo et al. (2018) highlighted the importance of the self-determination theory in supporting co-design activities. They proposed that the participants of a co-design process are motivated to contribute more and even work outside their comfort zone if their psychological needs for autonomy, competence, and relatedness are met. This finding was supported by Lindblom et al.'s (2021) work which demonstrated that acknowledgement of participants' insights motivates them to contribute more, even if they do not fully understand their role as co-designers.

Based on the aforementioned studies, we found it imperative to understand how interpersonal experiences between the designer and his co-designers affect the development process. The questions that drove this study were: (i) what does the process of co-designing an AR mobile application look like? and (ii) what are the experiences of a system designer in mediating the co-design process with non-designers?

\section{Method}

Because a system developer plays a vital role in translating ideas into viable concepts, this study focused on the experiences and roles of Riz, a system developer who was involved in the co-design and development of an AR mobile application for the study of the Central Nervous System. The co-design involved academic members of FMHS UPM, whom we regard as non-designers in this paper. Eliciting his experiences may offer insights into the value of co-design and the complex role of designers in mediating the design process with non-designers, all of which can lead to feasible project development.

As proposed by Merriam and Tisdell (2015), we adopted the qualitative research approach to gain an in-depth understanding of Riz's interpretation and meaning ascription surrounding his experiences in mediating the co-design process. Semi-structured interviews and observations were conducted while documents were analyzed to provide rich and descriptive findings (Fraenkel et al., 2011). Prior to data collection, the system developer gave his consent to participate; the name 'Riz' is a pseudonym to safeguard and protect his anonymity.

A total of three audio-recorded interviews with Riz, each lasting between 60 and 90 minutes, were transcribed. We read and re-read the transcripts to familiarize ourselves with the data (Creswell \& Poth, 2016). Meanwhile, observations were carried out each time Riz had meetings with the academic members of the FMHS. We observed a total of four meetings utilizing the non-participatory technique (Patton, 2002), as we wished to keep the co-design process as organic as possible without tampering with it. Field notes were taken during the observations. Finally, the documents we collected were screenshots of the AR mobile application, flowcharts, wireframes, and user interfaces provided by Riz, the system developer. Numerous memos were written as a pre-analysis of the interview transcripts, field notes, and documents before we began open coding (Charmaz, 2006).

\section{Results and Discussions}

We picture Riz as a fully dedicated and inspiring young man. He is knowledgeable and had over three years of experience in software development at the time of this study. He was the only system developer involved in the co-design process for the AR mobile application project. Having no medical education background, Riz depended on the insights of the FMHS academic members to understand latent users' needs. 


\subsection{The Process of Co-designing an AR Mobile Application}

In this section, we discuss our findings for the first question - what does the process of co-designing an AR mobile application look like? The themes that emerged suggest five phases in the process of developing an AR mobile application: i) planning, (ii) analysis, (iii) design, (iv) implementation, and (v) maintenance. These phases mimic the Waterfall Model, which is one of the Software Development Life Cycle (SDLC) models commonly used in the process of creating computer systems and software in the industrial and engineering fields (Thayer \& Yourdon, 1997). This is evidence that the system developer adhered to a specific SDLC model to work systematically towards the targeted output.

\subsubsection{Planning Phase}

The co-design process commenced when the system developer received requests from the academic members of the FMHS to design and develop an AR mobile application for the teaching and learning of the Central Nervous System. This phase set the foundation for the mobile app to be developed. At this point, Riz gathered input from his co-design partners, namely the FMHS academic members, specifically in identifying and analyzing the problems that occur in the medical classroom. The forthcoming excerpt explains the process:

At this phase, the stated problem was that the medical students need a model of the human brain when they want to study about the central nervous system. Because of the limited availability of this model, they could only use the model in the laboratory. They could not learn outside the laboratory as the model was not there. The purpose of this app is to provide them access to a model of the brain when they want to learn anytime. (Interview 1)

In this phase, Riz highlighted the importance of identifying the requirements of the user. He added that the dynamics of user requirements have a follow-up impact on learners' and educators' intuition when using the application. Therefore, both parties involved in the co-design process had to resolve any discrepancies that may arise:

In the planning phase, for me, user requirements are very important. The requirements show what the problem is, what learners' needs must be addressed, and what educators want. This is why I held several meetings with the FMHS team to clarify the requirements. They also required my expertise to explain the available technology and how to integrate it with the proposed application. (Interview 2)

System feasibility is the first phase of the Waterfall Model (Boehm et al., 1976). Focusing on the development proposal, this phase included the project's timeline, estimation plan, and communication plan. As it is the most crucial part of a system's development, it necessitated creating Gantt charts and flow charts from the beginning of the development until the implementation phase. Riz also utilized an instant group-messaging platform to facilitate swift communication between him and his non-designer co-design partners. Unanimous understanding and approval of all the co-designers ensured time efficiency. Figure 1 illustrates the flow chart of the AR mobile application project's planning.

\subsubsection{Analysis Phase}

The system developer then proceeded to the second phase - analysis. In this phase, Riz had to guide his co-designers in determining the necessary requirements for development. From the design jams he had with the academic members of the FMHS, they managed to consolidate a detailed outline on how the system should function along with its objectives, specifications, and application requirements. At the end of the final design jam, he collected the requirements from his co-design partners, as described in the following excerpt:

The FMHS academic members provided me with the user requirements, where the objective of the app, the justification for its development, and the required function of the app were consolidated in a single document. They also provided a draft interface on how the app should look and function (Interview 1)

A market search was deemed important at this stage to prevent redundancy in application functionality as well as to add novelty to the proposed mobile application. The following excerpt explains this step:

In this phase, I was required to explore various types of articles and journals. I also reviewed some applications in the current market. This gave me more information on how the proposed app should be, [such as] its design interface and content. (Interview 1)

Additionally, Riz emphasized other areas that needed equal attention, which were user scope, content scope, and system scope. Interestingly, his co-design partners were involved only in the user and content scope. The forthcoming interview excerpt explains this:

Based on my previous experience, when I need to design a new system, I normally divide it into three scopes. First is the user scope, then content scope and system scope. For the user scope, I need to analyze 
the user requirements given by the medical faculty. Then in the content scope, I look at the storyboard provided, and the system scope is where I prepare a design for the system and later present it to the stakeholder. (Interview 2)

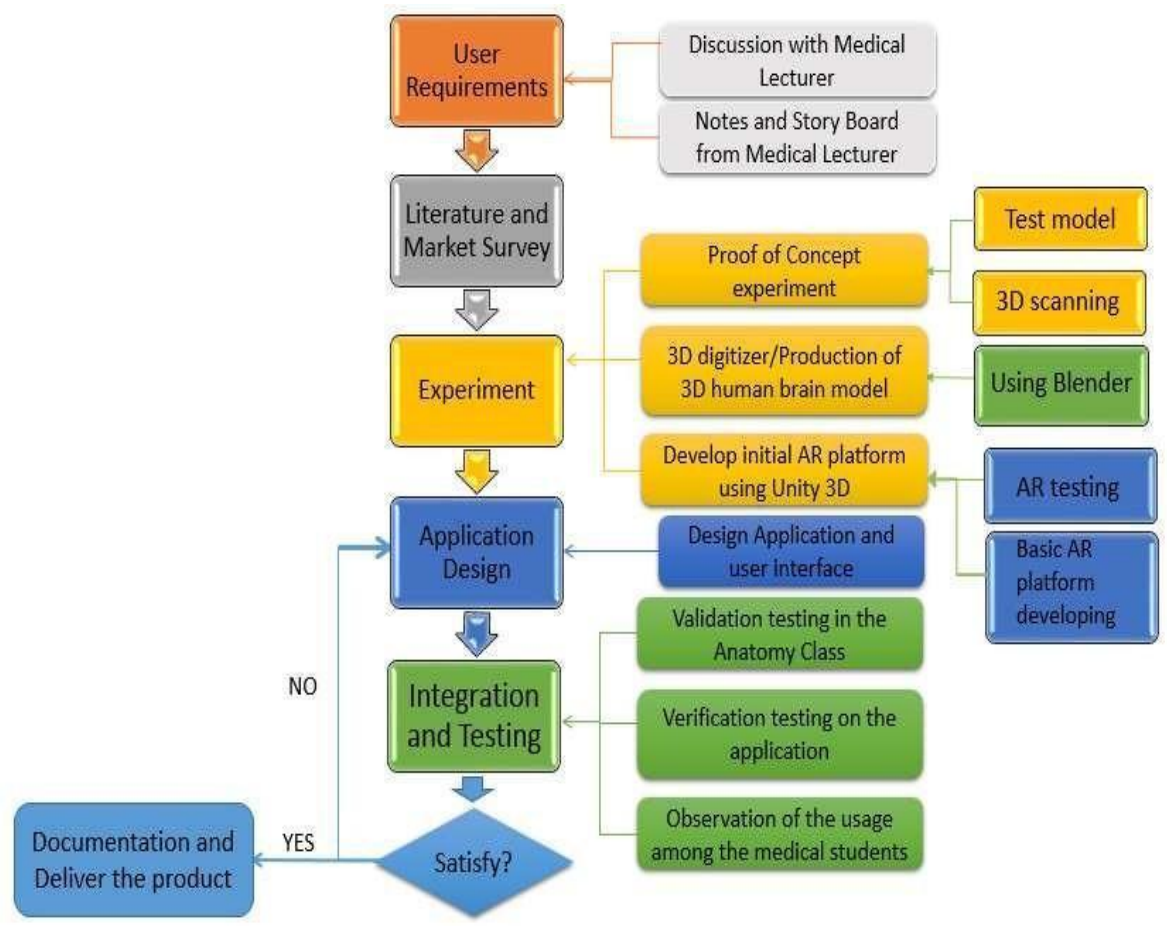

Figure 1. Flowchart of the AR mobile application project development plan

Riz also emphasized the technique used to ascertain the requirements given by his co-design partners. In this project, Riz employed an observational ethnographic approach to examine the current learning process and existing software system using AR technology. He stated that:

[...] traditional method is when I observed the current learning process in the classroom, and for application review, I analyzed and compared the strengths and weaknesses of each app related to this project. For example, the applications that I reviewed were Chemistry 101, AR flashcards, and the Octagon humanoid. Based on these apps, I looked into the system, interface, and function of the app. (Interview 3)

\subsubsection{Design Phase}

In the design phase, Riz worked on the storyboard to translate the requirements outlined by the academic members of the FMHS into a viable document. The storyboard served as their point of reference in understanding the architecture of the AR mobile application and its fulfillment of his co-design partners' requirements. Although Riz worked on the storyboard by himself, collaboration in this scenario took the form of sharing the storyboard with the academic members, who then verified or rectified the storyboard.

In the design phase, I created a storyboard like a blueprint which follows the requirements outlined by the medical faculty members. From their complex requirements, I simplified it into a storyboard. I also made a draft interface for the design application - around 20 pictures of the application interface that represents how the application looks, its specific buttons, and how they interact with the content - then I presented it to the faculty members. (Interview 1)

\subsubsection{Implementation Phase}

The implementation phase focused on translating the abstract concepts into a tangible product. In this phase, Riz played three important roles, namely programmer, interface designer, and tester specialist. Apart from this, the co-designer partners and Riz collaboratively worked on six key activities to complete the AR mobile application. The activities were modelling through the digitalization process, coding, video recording, testing, re-testing, and documentation. With the academic members, Riz took a digital photo of the real brain model from several angles to build a digital three-dimensional model for the AR experience. He worked on anchoring each picture, symbol, 
button, and model to specific actions; his co-design partners were not involved in this activity. He later proceeded to the video recording activity, about which he commented:

Based on the requirements agreed with the academic members, I was given a task to develop a video specifically for this app. I needed to set up a schedule with the medical academic members and do a video recording of an academic giving a lecture on the central nervous system." (Interview 3 )

Testing was then conducted to confirm if the application adheres to the users' needs and follows their plan. For this purpose, a series of validation sessions were conducted with the FMHS academic members who are subject-matter experts on the Central Nervous System. The participants contributed their feedback on ways to improve the mobile application, as explained below:

In the testing phase, I had to do a validation process to confirm the function of the app tallied with the requirements of the stakeholders. The first step in the testing phase was validation with medical experts. They tried the app and then gave feedback on the function and content used. (Interview 3)

Additionally, a re-validation was carried out with medical students as they are the targeted users of the application. The interview excerpt below explains the activity:

After the testing process with the medical experts, I had to make some improvements. After one week of improvements, I did re-testing with 12 students from FMHS to get feedback from their perspective. (Interview 3)

Feedback from both sessions was then consolidated and taken into account when finalizing the AR mobile application. According to Riz, the implementation phase involved the most work compared to other phases:

For me, this phase was the longest and most difficult compared to other phases, because I took a longer time to find the right technique for the digitization process. I should have completed it in week 10; however, due to the difficulty in the digitization process, I completed it in week 13 as I only discovered the right technique then. (Interview 1)

\subsubsection{Maintenance Phase}

Maintenance is the final phase after the application was finalized. This phase is important in sustaining the optimum use of the application. However, the designer mentioned that the maintenance for the AR application was borne by his co-design partners, namely the academic members of the FMHS as he was engaged only as a freelancer and did not directly belong to the team.

\subsection{The System Developer's Experience in Mediating the Process of Co-design with Non-designers}

This section discusses the findings of the second research question - what are the experiences of a system developer in mediating the process of co-design with non-designers? The findings for this question echo the findings for the first one. Through our analysis, we found that Riz played an instrumental role throughout the process of mediating the designer and co-designers' (i) communication, (ii) commitment to building a cohesive working relationship as well as to the delivery and quality of the AR mobile application, and (iii) motivation in working together.

\subsubsection{Communication}

Communication was one of the main factors behind the successful AR mobile app co-design process that led to its feasible project development. Without effective communication, Riz mentioned that several conflicts would have arisen, such as misunderstandings about the responsibility of each co-designer's role. His effort in creating a communication platform via an instant messaging system accessible on each member's smartphone made the process swift. According to Riz, the positive impact of well-managed communication did not only create a meaningful co-design experience, but more importantly, led to the success of the AR mobile application's development. This aligns with Waller et al.'s (2016) assertion that the clear understanding of roles, responsibilities, and information design is a key success factor in shaping a culture of cooperation and providing solutions to common communication problems.

3.2.2 Commitment to Building a Cohesive Working Relationship and to the Delivery and Quality of the AR Mobile Application

The aggregated comments given by Riz gave a clear message that every member of the co-designer team had to fully commit to ensuring the project was delivered on time. Each person's commitment to success was essential during the development process. The system developer mentioned that following best practices to manage delivery time was just as important as preserving the quality of the AR mobile application. This demonstrates the 
level of professionalism and commitment necessary for creating a quality product. Riz further underscored that maintaining healthy and robust bonds with his co-designers was vital in building a working relationship, despite some disagreements along the way. By doing so, he initiated a higher level of volition among his co-designers, which led to their increased engagement and quality contributions (Dent-Spargo, 2018; Lindblom et al., 2021).

\subsubsection{Motivation in Working with Co-designers}

Through the memos garnered from the observations, we detected a sense of pride and accomplishment among the co-designers when the AR mobile application was completed. In some instances, it was evident that the co-designers had genuine affection for one another; this sense of friendship is what made their working relationship stronger. According to Dent-Spargo (2018), participants in a co-design process are usually motivated to contribute above and beyond their duties when their psychological needs for autonomy, competence, and relatedness are met. Recent work by Lindblom et al. (2021) resonates with these findings by adding that although participants may not fully understand their participatory role, they are more motivated to contribute when their insights are valued. Indeed, Riz said that the moral support, affection, and kindness shown by his co-designer partners made him enjoy his work more.

The system developer's experience and roles in mediating the process of co-design with non-designers are explained in Figure 2.

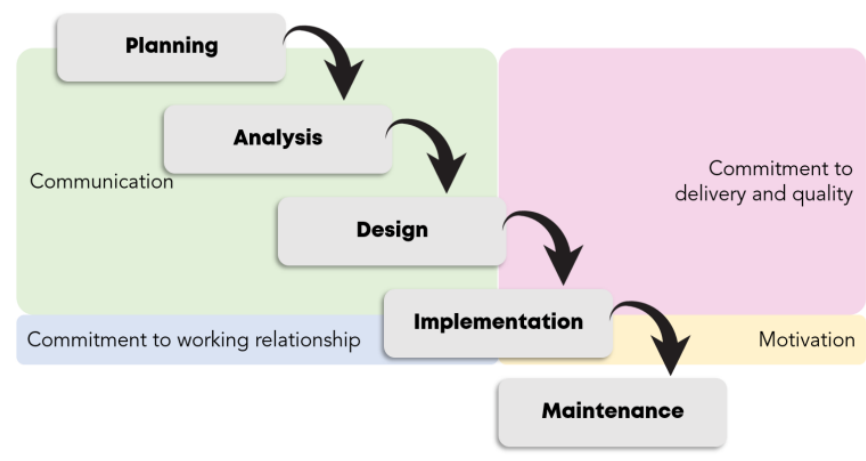

Figure 2. System developer's role in mediating the co-design process through the Waterfall Model.

\section{Concluding Remarks}

This research found that it is imperative for a system developer to adhere to a certain SDLC model to develop an AR mobile application. In this study, the system developer followed the Waterfall Model to assist him throughout the co-design process that he mediated with his non-designer co-design partners, namely the academic members of the FMHS. We found that the phases of the Waterfall Model reciprocated one another in a non-linear method throughout the co-design process, as the process involved several negotiations between the system developer and his co-designers. This led to several revisitations to the phases of the model, namely planning, analysis, design, implementation, and maintenance. Because the system developer had non-designer counterparts in the co-design process, his role was vital in ensuring the success of the AR mobile application. Consequently, communication, commitment, work enjoyment, and satisfaction emerged as important factors that he needed to mediate in ensuring the feasibility of the project. This was embodied by the co-designers' motivation and willingness to contribute during the sessions and sustain their involvement throughout the process. Since the co-designers were also users of the AR mobile application, they showed great responsibility in expressing themselves creatively as they had a sense of ownership over their contributions. Listening to, understanding, and valuing the non-designers was important for the system developer in mediating the co-design process as well, so that the co-design partners knew that they had control over what they envisioned to achieve. Overall, since system developers possess more design knowledge, their role in finding the right balance and mediating the co-design process with their non-designer partners is instrumental in ensuring the success of any system development. We conclude that system development through co-design is a concerted effort that involves dynamics of power between system developers and their non-designer counterparts. In this regard, this study has shed light on the roles of the system developer in the co-design process in each phase of the Waterfall Model under the SDLC.

\section{References}

Ashkenas, R. (2015, April 20). There's a Difference between Cooperation and Collaboration. Retrieved from 
https://hbr.org/2015/04/theres-a-difference-between-cooperation-and-collaboration.

Boehm, B. W., Brown, J. R., \& Lipow, M. (1976). Quantitative evaluation of software quality. In R. T. Yeh, \& C. V. Ramamoorthy (Eds.), Proceedings of the 2nd international conference on Software engineering ( $\mathrm{pp}$. 592-605). IEEE Computer Society Press.

Boyd, R. A., \& Peters, M. (2009). Using MAPP to connect communities: One county's story. The Health Educator, 41, 77-84.

Warburton, J., Everingham, J., Cuthill, M., \& Bartlett, H. (2008). Achieving effective collaborations to help communities age well. The Australian Journal of Public Administration, 67, 470-482. https://doi.org/10.1111/j.1467-8500.2008.00603.x

Brown, L. D., Feinberg, M. E., \& Greenberg, M. T. (2012). Measuring Coalition Functioning: Refining Constructs Through Factor Analysis. Health Education \& Behavior, 39(4), 486-497. https://doi.org/10.1177/1090198111419655

Ceraulo, S. C. (2005). Benefits of upgrading to an LMS. Distance Education Report, 9(9), 6-7.

Charmaz, K. (2006). Constructing grounded theory: A practical guide through qualitative analysis. Sage Publications.

Creswell, J. W., \& Poth, C. N. (2017). Qualitative inquiry and research design: Choosing among five approaches. Sage Publications.

Darras, K. E., Spouge, R., Hatala, R., \& Nicolaou, S. (2019). Integrated virtual and cadaveric dissection laboratories enhance first year medical students' anatomy experience: A pilot study. BMC Medical Education, 19, 366. https://doi.org/10.1186/s12909-019-1806-5

Dent-Spargo, R. (2018). Using Self-Determination Theory to Support Co-Design Activities. In A. Piotrkowicz, R. Dent-Spargo, S. Dennerlein, I. Koren, P. Antoniou, P. Bailey, ... C. Pahl, (Eds.). CEUR Workshop Proceedings. CC-TEL 2018: Co-Creation in the Design, Development and Implementation of TechnologyEnhanced Learning. 03 Sep 2018, Leeds, UK.

Dimopoulos-Bick T., Dawda P, Maher L, Verma R, Palmer V. (2018). Experience-based co-design: Tackling common challenges. The Journal of Health Design, 3(1), 86-93. https://doi.org/10.21853/JHD.2018.46

Fraenkel, J. R., Wallen, N. E., \& Hyun, H. H. (2011). How to design and evaluate research in education. McGraw-Hill.

Grant, A. M., \& Shin, J. (2012). Work motivation: Directing, energizing, and maintaining effort (and research). In R. M. Ryan (Ed.), Oxford Handbook of Motivation (pp. 505-519). Oxford, UK: Oxford University Press.

Jones, R. B., Stallard, P., Agha, S. S., Rice, S. Werner-Seidler, A., Stasiak, K., ... Merry, S. (2020). Practitioner review: Co-design of digital mental health technologies with children and young people. The Journal of Child Psychology and Psychiatry, 61(8), 928-940. https://doi.org/10.1111/jcpp.13258

Dijck, J. Van. (2000). Digital Cadavers: The visible human project as anatomical theater. Studies in History and Philosophy of Biology \& Biomedical Science, 31(2), 271-285

Keys, Y., Silverman, S. R., \& Evans, J. (2016). Identification of Tools and Techniques to Enhance Interdisciplinary Collaboration during Design and Construction Projects. Health Environments Research \& Design Journal, 10(5), 28-38. https://doi.org/10.1177/1937586716684135

Lindblom, S. Flink, M., Elf, M., Laska, A. C., von Koch, L., \& Ytterberg, C. (2021). The manifestation of participation within a co-design process involving patients, significant others and health-care professionals. Health Expectations, 24(3), 905-916. https://doi.org/10.1111/hex.13233

Marek, L. I., Brock, D. J. P., \& Savla, J. (2015). Evaluating collaboration for effectiveness conceptualization and measurement. American Journal of Evaluation, 36(1), 67-85.

Merriam, S. B., \& Tisdell, E. J. (2015). Qualitative research: A guide to design and implementation. John Wiley $\&$ Sons.

Molnar \& Palmas (2021). Dissonance and diplomacy: Coordination of conflicting values in urban co-design. Co-Design, 1-15. https://doi.org/10.1080/15710882.2021.1968441

Mulvale, G., Green, J., Miatello, A., Cassidy, A. E., \& Martens, T. (2020). Finding harmony within dissonance: Engaging patients, family/caregivers and service providers in research to fundamentally restructure relationships through integrative dynamics, Health Expectations 24(S1), 147-160. 
https://doi.org/10.1111/hex.13063

O’Hare, J., Dekoninck, E., Mombeshora, M., Martens, P., Becattini, N., \& Boujut, J. (2020). Defining requirements for an Augmented Reality system to overcome the challenges of creating and using design representations in co-design sessions. CoDesign, 16(2), 111-134. https://doi.org/10.1080/15710882.2018.1546319

Patton, M. Q. (2002). Qualitative evaluation and research methods. Sage.

Sanders, L., \& Stappers, P. J. (2014). From designing to co-designing to collective dreaming: Three slices in time. Interactions, 21(6), 24-33. https://doi.org/10.1145/2670616

Saraiva, R., \& Iglesias, F. (2016). Cooperation under pressure: Time urgency and time perspective in social dilemmas. Time \& Society, 25(2), 393-405. https://doi.org/10.1177/0961463X15577271

Trischler, J., Pervan, S. J., Kelly, S. J., \& Scott, D. R. (2017). The value of codesign: The effect of customer involvement in service design teams. Journal of Service Research, 21(1), 75-100. https://doi.org/10.1177/1094670517714060

Van Lange, P. A. M., Joireman, J., Parks, C. D., \& Van Dijk, E. (2013). The psychology of social dilemmas: A review. Organizational Behavior and Human Decision Processes, 120(2), 125-141. https://doi.org/10.1016/j.obhdp.2012.11.003

Waller, R., Waller, J., Haapio, H., Crag, G., \& Morrisseau, S. (2016). Cooperation through clarity: Designing simplified contracts. Journal of Strategic Contracting and Negotiation, 2(1-2), 48-68.

Yousuf, I. M., Anwar, M. N., \& Sarwar, M. (2008). Perceptions of course coordinators and course writers for developing distance learning material. Turkish Online Journal of Distance Education, 9(2), 123-137.

Zhu, Y. Q., Gardner, D. G., \& Chen, H. G. (2016). Relationships between Work Team Climate, Individual Motivation, and Creativity. J. of Management, 44(5), 2094-2115. http://doi.org/10.1177/0149206316638161

\section{Copyrights}

Copyright for this article is retained by the author(s), with first publication rights granted to the journal.

This is an open-access article distributed under the terms and conditions of the Creative Commons Attribution license (http://creativecommons.org/licenses/by/4.0/). 